DOUGLAS RICARDO SLAUGHTER NYIMI

COMPUTABILIDADE E LIMITES DA MATEMÁTICA DAS TEORIAS FÍSICAS: APLICAÇÕES EM SISTEMAS ELÉTRICOS DE POTÊNCIA 
DOUGLAS RICARDO SLAUGHTER NYIMI

\section{COMPUTABILIDADE E LIMITES DA MATEMÁTICA DAS TEORIAS FÍSICAS: APLICAÇÕES EM SISTEMAS ELÉTRICOS DE POTÊNCIA}

Tese apresentada à Escola Politécnica da Universidade de São Paulo para a obtenção do título de Doutor em Engenharia.

Área de Concentração:

Sistemas de Potência

Orientador: Prof. Livre-Docente

José Aquiles Baesso Grimoni 
Este exemplar foi revisado e alterado em relação à versão original, sob responsabilidade única do autor e com a anuência de seu orientador.

São Paulo, ........ de outubro de 2011.

Assinatura do autor

Assinatura do orientador

FICHA CATALOGRÁFICA

Slaughter, Douglas Ricardo Nyimi

Computabilidade da matemática das teorias físicas: aplicações em sistemas elétricos de potência / D.R.N. Slaughter. -ed.rev.-- São Paulo, 2011.

$160 \mathrm{p}$.

Tese (Doutorado) - Escola Politécnica da Universidade de São Paulo. Departamento de Engenharia de Energia e Automação Elétricas.

1. Sistemas elétricos de potência 2. Sistemas de controle 3. Teoria da computação 4. Teoria do caos 5 . Física matemática 6. Matemática da computação I. Universidade de São Paulo. Escola Politécnica. Departamento de Engenharia de Energia e Automação Elétricas II. t. 


\section{DEDICATÓRIA}

A Jennifer

A Neide 


\section{AGRADECIMENTOS}

Ao Prof. Dr. José Aquiles Baesso Grimoni pela amizade, convivência, auxílio, confiança e orientação dedicada.

Ao Prof. Dr. Gildo Magalhães dos Santos Filho pelas sugestões, boa vontade, conversas e por uma recomendação crucial: "comece pelo meio".

Ao Prof. Dr. João José Neto pelas conversas esclarecedoras sobre computabilidade, orientações com relação à importância do formalismo matemático, pelo curso de fundamentos da computação e por sua detalhada avaliação de uma versão da tese. Foram contribuições decisivas.

Ao Prof. Dr. Lineu Belico dos Reis pela ajuda, esclarecimento de dúvidas e avaliação de versões preliminares do trabalho.

Ao Prof. Dr. Luiz Barco pelas sugestões e sábias palavras com relação à vida.

Ao Prof. Dr. Osvaldo Frota Pessoa Jr. e ao grupo REDUX (Fábio Gatti, Fábio Leite, Luiz Rigolin, Nelson Bejarano e Yara Kulaif). As apresentações e debates do grupo foram fundamentais para a estruturação desta tese.

Ao Prof. Dr. Walter Kaiser pela boa vontade e colaboração. Graças a sua ajuda, este doutorado foi possível.

A Jennifer, minha noiva, meu Sol de todos os dias, o Norte de minha vida.

A Neide, minha mãe, por seu amor e respaldo incondicionais durante toda minha vida.

A Christian, meu irmão e companheiro de viagem, pela convivência, conversas sobre todos os assuntos e ajuda na busca de sentidos para a vida.

Aos meus tios Dirce, Helena e Edson pelo interesse e incentivo. 
Ao grande amigo Alexandre Coutinho Lisboa pelas estimulantes conversas e sua contagiante paixão pela busca do saber.

À Escola Politécnica e à Universidade de São Paulo por proporcionar a oportunidade de realizar um doutorado de alto nível de forma gratuita.

Ao Conselho Nacional de Desenvolvimento Científico e Tecnológico (CNPq) pela bolsa de estudos, que proporcionou as condições financeiras para a elaboração deste trabalho com tranquilidade. 
"There are more things in heaven and earth, Horatio,

Than are dreamt of in your philosophy",

Hamlet

"To a man with a hammer, everything looks like a nail"

Mark Twain

"Eu prefiro ser essa metamorfose ambulante

Do que ter aquela velha opinião formada sobre tudo"

Raul Seixas

\footnotetext{
${ }^{1}$ Esta frase é dita por Hamlet a Horácio, no ato I, cena V, da peça Hamlet de William Shakespeare (1564-1616). A expressão 'your philosophy' se refere à filosofia natural (que, a grosso modo, corresponde às ciências naturais atuais) das quais Horácio era um estudioso.
} 


\section{RESUMO}

Apesar dos modelos usados em engenharia serem, em sua maioria, reconhecidamente aproximados, acredita-se que a matemática usada na física e nos próprios modelos é infinitamente precisa e que tais teorias físicas poderiam prever completamente qualquer evento relacionado às variáveis equacionadas. No limite, seria possível prever o estado do "universo" em qualquer instante, crença esta chamada de determinismo. Claro está que essa pretensão é apenas de princípio, sendo impossível na prática. No entanto, pesquisas sobre os fundamentos da matemática e outras teorias matemáticas desenvolvidas no século XX sugerem que a matemática (e, consequentemente, a física) teria certos limites inerentes. A análise feita nesta tese fundamenta seus argumentos na Teoria das Funções Recursivas e Computabilidade Efetiva e na Teoria do Caos Determinístico. O objetivo principal é tratar de apurar a existência de limites inerentes e como tais limites se aplicariam aos sistemas elétricos de potência (mais especificamente nos tópicos fluxo de carga, transitórios eletromecânicos, transitórios eletromagnéticos e eletrônica de potência) e à engenharia de controle.

Palavras-chave: Computabilidade, Turing, Caos, Matemática, Física, Modelos matemáticos, Sistemas elétricos de potência, Determinismo, Laplace, Limitações. 


\begin{abstract}
Although the models used in engineering are, in most cases, admittedly approximated, it is believed that the Mathematics used in Physics and in these models, is infinitely precise and that such physical theories could fully predict any event related to variables in equations. In the limit, it would be possible to predict the state of the "universe" at any moment, this belief is called determinism. It is clear that this claim is only in principle, impossible in practice. However, research on the foundations of Mathematics and other mathematical theories developed in the $20^{\text {th }}$ century suggest that the Mathematics (and hence Physics) would have certain inherent limitations. The analysis made in this thesis has the arguments based on the Theory of Recursive Functions and Effective Computability and the Theory of Deterministic Chaos. The main objective is to find out the existence of inherent limits and how these limits could be applied to electric power systems (more specifically to the topics load flow, electromechanical transient and electromagnetic transient and power electronics) and control engineering.
\end{abstract}

Keywords: Computability, Turing, Chaos, Mathematics, Physics, Mathematical models, Power electric systems, Determinism, Laplace, Limitations. 


\section{LISTA DE ILUSTRAÇÕES}

Figura 1 - Diagrama de Venn de alguns conjuntos numéricos infinitos........................... 30

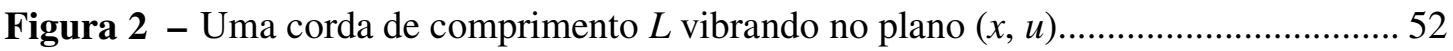

Figura 3 - Uma solução da equação de onda unidimensional para instantes sucessivos discretos.

Figura 4 - Problema dos 3 corpos (uma partícula de poeira na órbita de dois planetas de massas iguais). A complexidade da dinâmica se deve ao caráter nãolinear das equações diferenciais

Figura 5 - Evolução temporal de uma das variáveis das equações diferenciais de Lorenz. Uma das curvas foi simulada com a condição inicial 0.506127 e, a outra, com a condição inicial 0.506. Elas coincidem no princípio, mas divergem completamente logo depois

Figura 6 - A curva apresenta "padrões" aperiódicos e amplitude limitada

Figura 7 - Uma representação pictórica de Euclides de Alexandria ( 325 a. C.- 265

a. C.), autor de "Os Elementos"

Figura 8 - Fluxograma do algoritmo de Euclides.

Figura 9 - Fita da máquina de Turing

Figura 10- Máquina de Turing "física”.

Figura 11- Máquina de Turing $M_{e}$ representada por um diagrama de estados.

Figura 12- Distribuição no $\mathbb{R}$ de números computáveis (pontos laranjas) e incomputáveis (plano azul)

Figura 13- Uma onda de tensão que é função de $(x$-vt) é mostrada para valores de $t$ iguais a $t_{1}$ e $t_{2}$

Figura 14- Diagrama do circuito de Chua com destaque ao bipolo não-linear...

Figura 15- Grandeza elétrica $i_{N R}\left(V_{C_{1}}\right)$ do bipolo não-linear ("piecewise linear") do circuito de Chua.

Figura 16- Evolução temporal da tensão no capacitor $C_{2}\left(V_{C_{2}}\right)$.

Figura 17- Circuito do conversor Cúk

Figura 18- Forma de operação do "Demônio de Laplace", 


\section{LISTA DE TABELAS}

Lista 2.1 - Bijeção entre o $\mathbb{N}$ e outro conjunto infinito.

Lista 2.2 - Tentativa de bijeção entre o $\mathbb{N}$ e o conjunto dos números irracionais entre

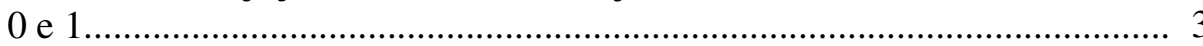

Tabela 3.1 - Classificação de problemas matemáticos e sua facilidade de solução por métodos analíticos................................................................................... 46

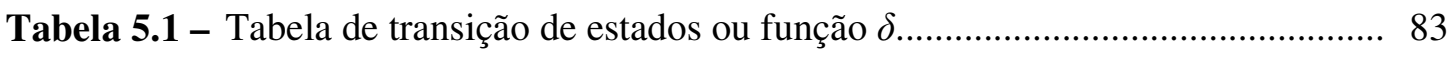

Lista 6.1 - Tentativa de bijeção entre todos os algoritmos (máquinas de Turing (MT)) possíveis e o conjunto dos números irracionais entre 0 e 1 ......................... 90

Tabela 6.1 - Diferentes classificações dos números reais e sua distribuição em termos de computabilidade................................................................................. 96

Tabela 6.2 - Diferentes tipos de conjuntos e sua distribuição em termos de computabilidade................................................................................... 99

Tabela 6.3 - Diferentes tipos de funções e sua distribuição em termos de computabilidade............................................................................... 101

Lista 6.2 - Conjunto de todas as cadeias possíveis $\left(\Sigma^{*}\right)$ do alfabeto $\Sigma=\{a, b, c, d, \ldots$,

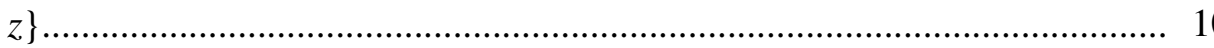

Tabela 6.4 - Diferentes aspectos das linguagens formais e sua computabilidade.

Tabela 7.1 - Computabilidade das equações diferenciais, das condições iniciais, das soluções e de valores reais particulares 


\section{LISTA DE ABREVIATURAS E SIGLAS}

$\begin{array}{ll}\text { DSCI } & \text { Dependência Sensitiva das Condições Iniciais } \\ \text { EDO } & \text { Equação Diferencial Ordinária } \\ \text { EDP } & \text { Equação Diferencial Parcial } \\ \text { EOM } & \text { Encyclopaedia of Mathematics } \\ \text { MDC } & \text { Máximo Divisor Comum } \\ \text { MT } & \text { Máquina de Turing } \\ \text { RPS } & \text { Regime Permanente Senoidal } \\ \text { SEP } & \text { Stanford Encyclopedia of Philosophy } \\ \text { UTM } & \text { Universal Turing Machine } \\ \text { ZFC } & \text { Teoria de conjuntos axiomatizada de Zermelo-Fraenkel acrescida do "axioma } \\ & \text { de escolha" (axiom of Choice) }\end{array}$




\section{SUMÁRIO}

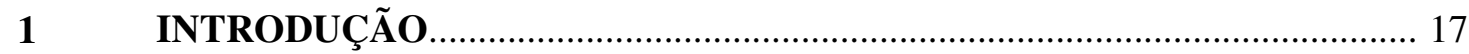

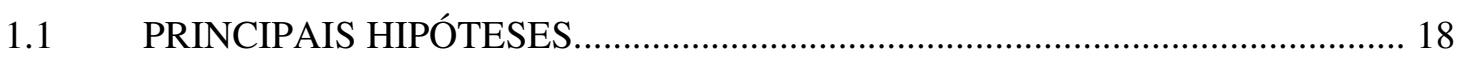

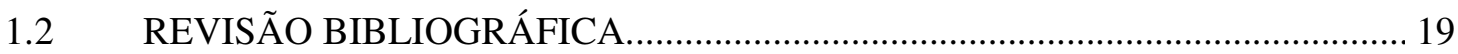

2 NOÇÕES SOBRE A TEORIA DE CONJUNTOS ………………………......... 22

2.1 A IMPORTÂNCIA DA TEORIA DE CONJUNTOS PARA A MATEMÁTICA.................................................................................. 22

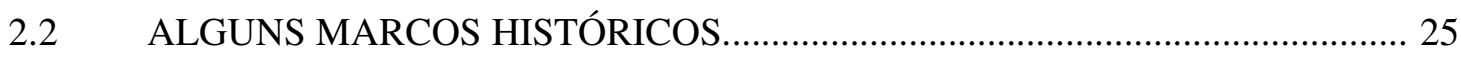

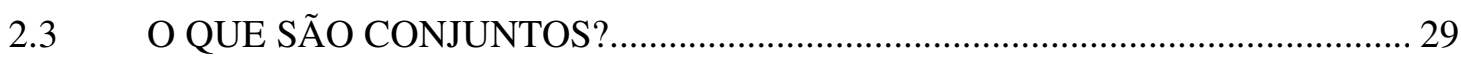

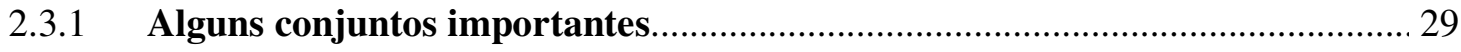

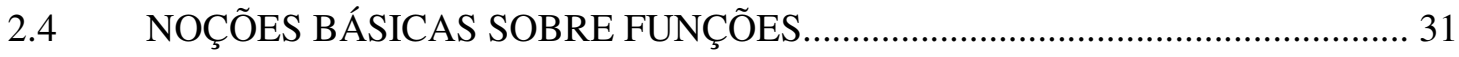

2.4.1 Definição de função.......................................................................................... 31

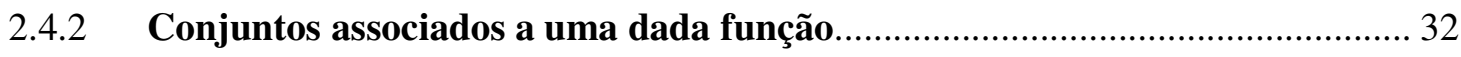

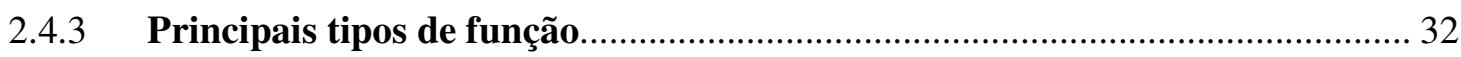

2.5 PRINCIPAIS CONCEITOS E PROPRIEDADES............................................. 34

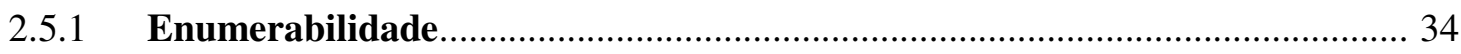

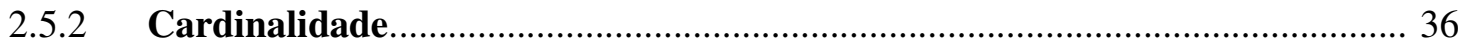

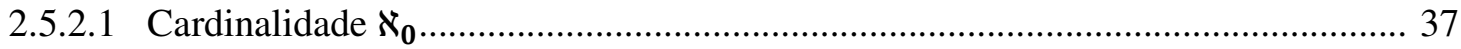

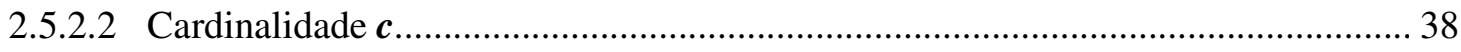

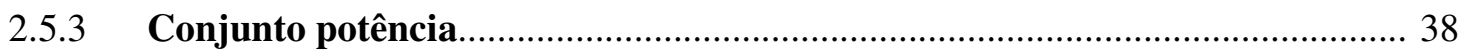

3 EQUAÇÕES DIFERENCIAIS............................................................ 41

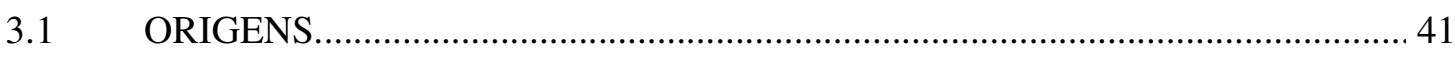

3.2 TIPOS PRINCIPAIS DE EQUAÇÕES DIFERENCIAIS....................................... 43

3.2.1 Quanto ao número de variáveis independentes................................................ 43

3.2.1.1 Equações diferenciais ordinárias (EDOs) ................................................................ 43

3.2.1.2 Equações diferenciais parciais (EDPs) .................................................................. 44

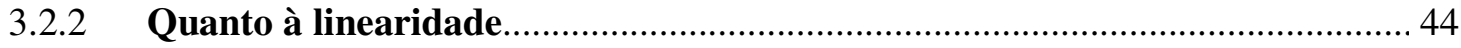

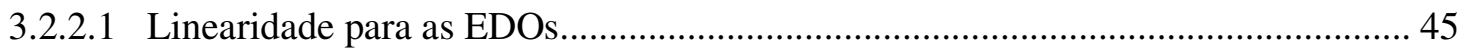

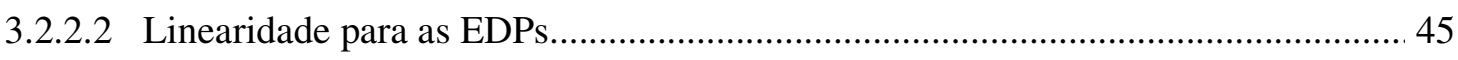

3.3 SOLUBILIDADE DAS EQUAÇÕES DIFERENCIAIS ......................................... 45

3.3.1 Teorema Fundamental das equações diferenciais............................................. 46 
3.3.1.1 Teorema Fundamental para as EDOs............................................................. 47

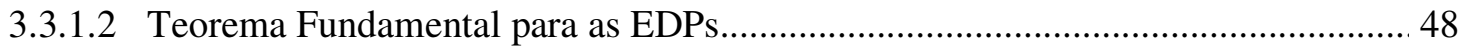

3.3.1.3 Exemplos de equações diferenciais onde a solução não existe ou não é única....... 48

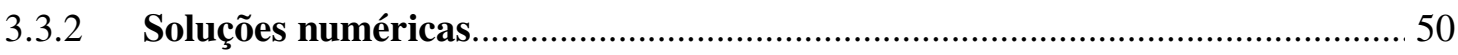

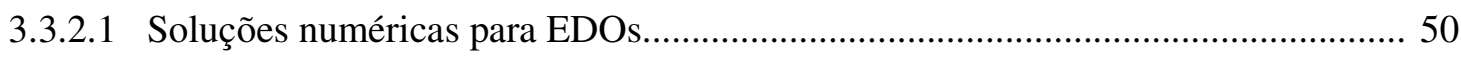

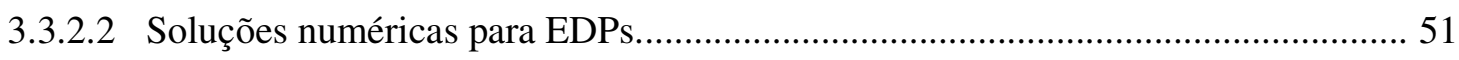

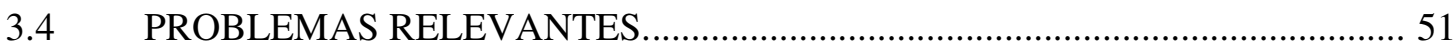

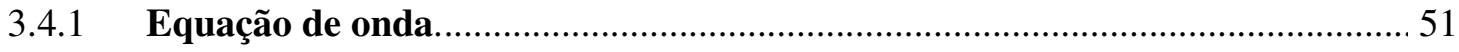

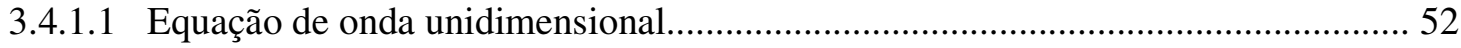

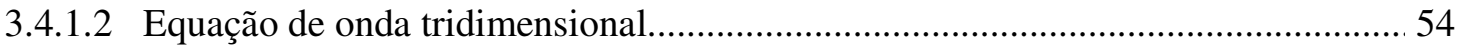

4 TEORIA DO CAOS DETERMINÍSTICO: NOÇÕES SOBRE SISTEMAS

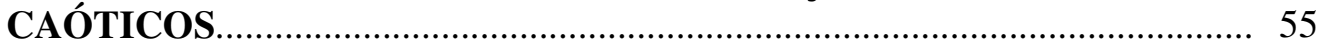

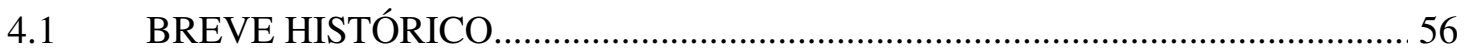

4.2 POR QUE O USO DO TERMO “DETERMINÍSTICO”?................................... 57

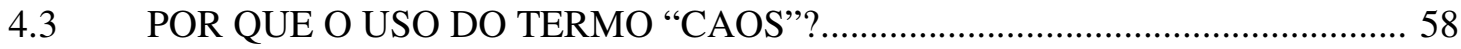

4.3.1 Definição de Dependência Sensitiva das Condições Iniciais............................ 60

4.3.2 Dinâmica caótica comparada a outras dinâmicas............................................. 62

4.4 DINÂMICA CAÓTICA E SUAS IMPLICAÇÕES NA FÍSICA............................ 64

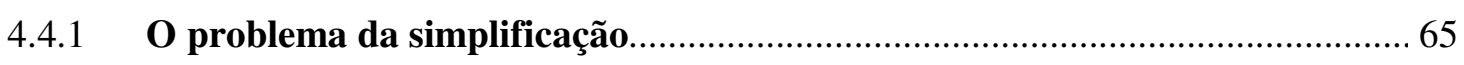

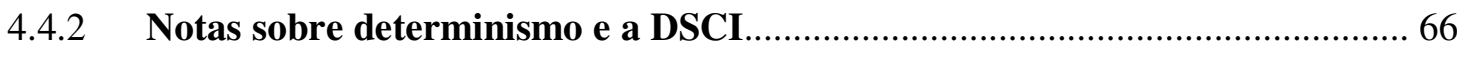

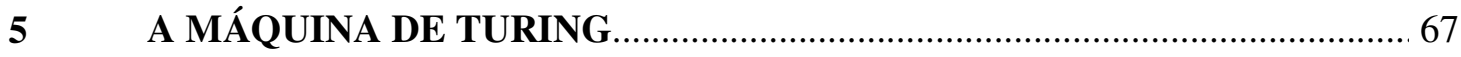

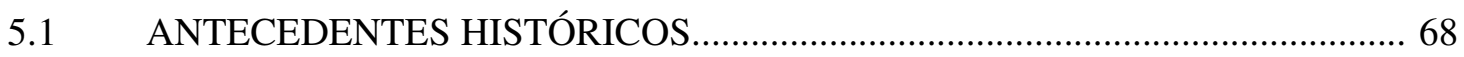

5.1.1 Pequena digressão sobre os sistemas formais axiomáticos................................ 68

5.1.2 A lógica dentro da crise da teoria de conjuntos.............................................69

5.1.3 Requisitos para o sucesso do programa formalista........................................ 71

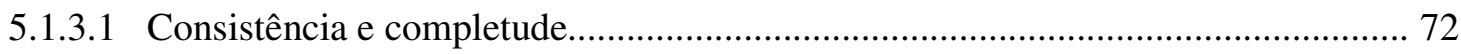

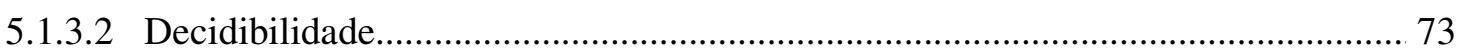

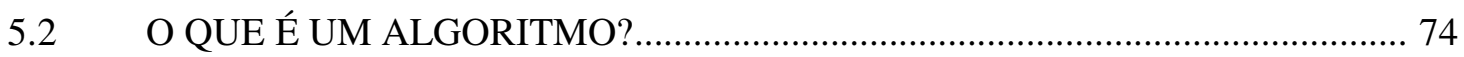

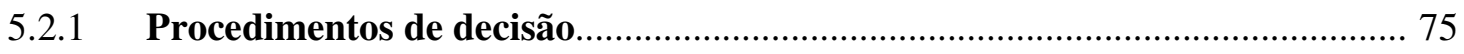

5.2.1.1 Procedimentos de decisão em engenharia elétrica.............................................. 76

5.3 PROBLEMA DE DECISÃO E A MÁQUINA DE

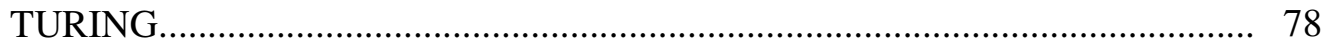

5.4 DUAS FORMULAÇÕES DA MÁQUINA DE TURING ................................... 80

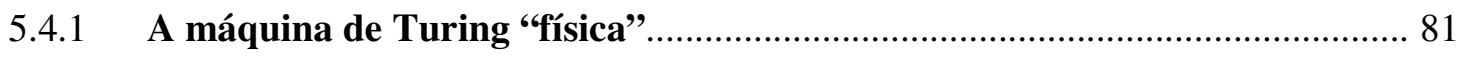




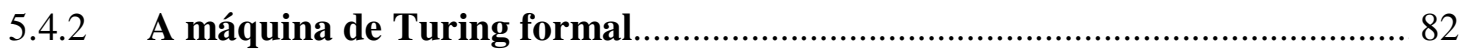

5.5 FUNCIONAMENTO DA MÁQUINA DE TURING....................................... 83

5.6 MÁQUINAS EQUIVALENTES E MÁQUINA DE TURING UNIVERSAL...... 84

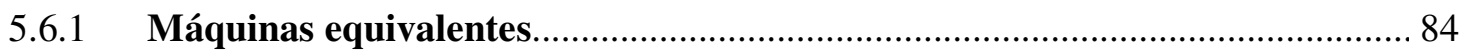

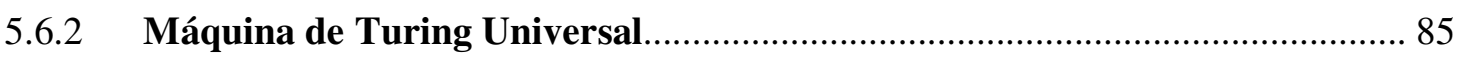

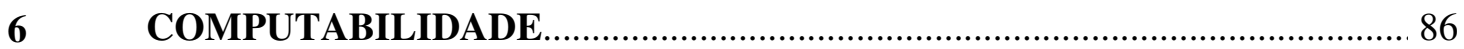

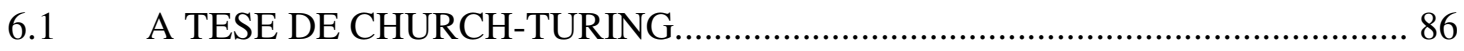

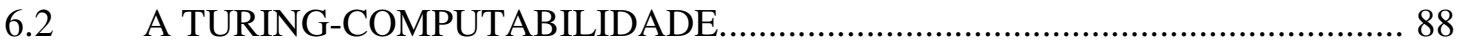

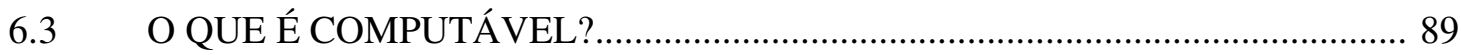

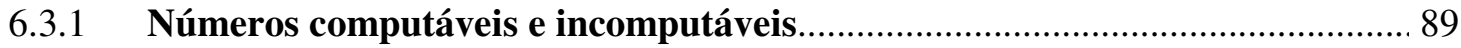

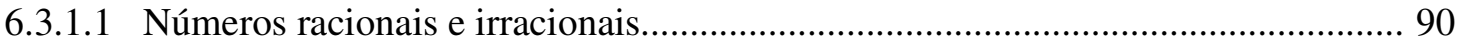

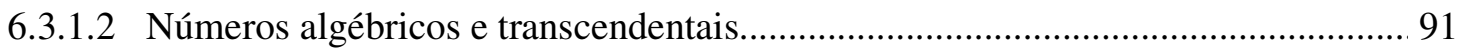

6.3.1.3 Números reais simplesmente normais e absolutamente normais........................... 93

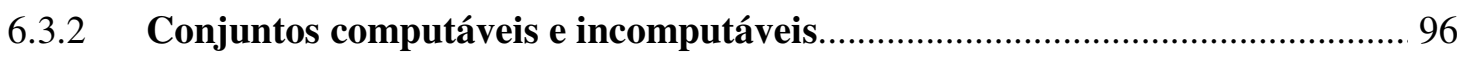

6.3.3 Funções computáveis e incomputáveis......................................................... 99

6.3.4 Tópicos de linguagens formais e sua computabilidade.................................. 102

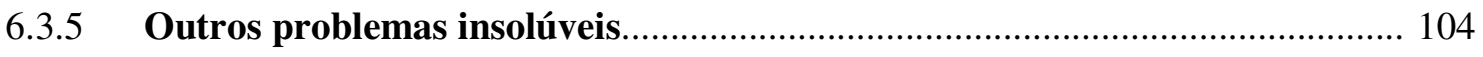

7 APLICAÇÕES EM SISTEMAS ELÉTRICOS DE POTÊNCIA E

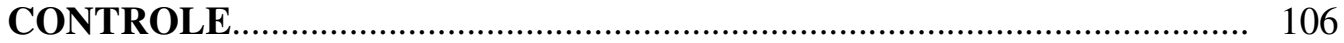

7.1 ASPECTOS MATEMÁtICOS GERAIS DAS FERRAMENTAS E MÉTODOS .................................................................................... 106

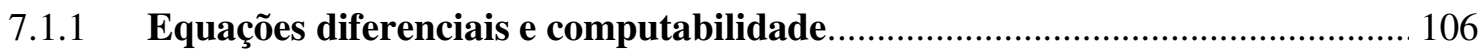

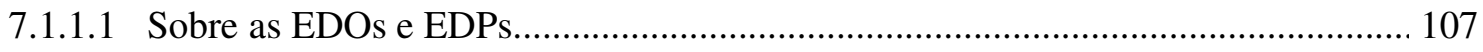

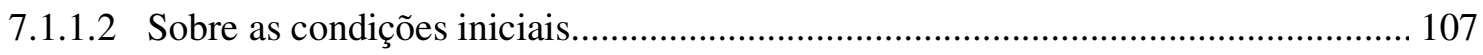

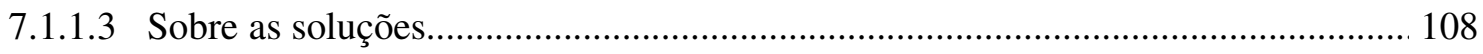

7.1.2 Métodos numéricos e computabilidade.......................................................... 109

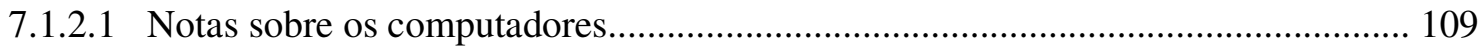

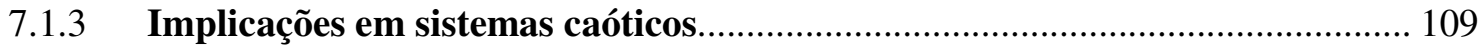

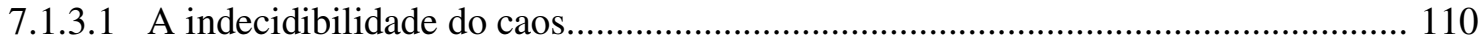

7.2 APLICAÇÕES EM SISTEMAS ELÉTRICOS DE POTÊNCIA........................ 111

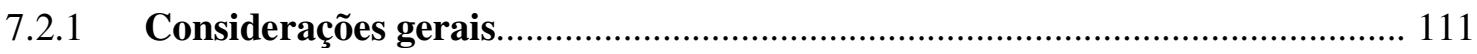

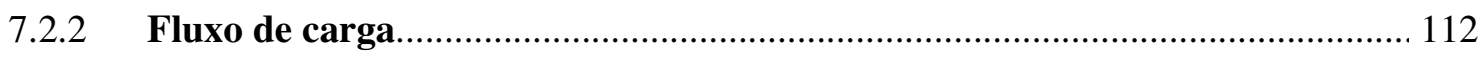

7.2.3 Transitórios eletromecânicos...................................................................... 113

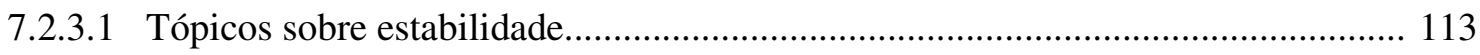




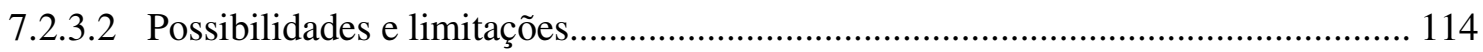

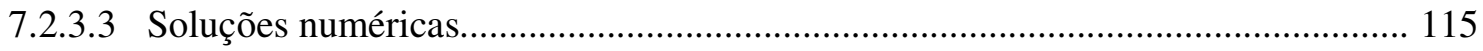

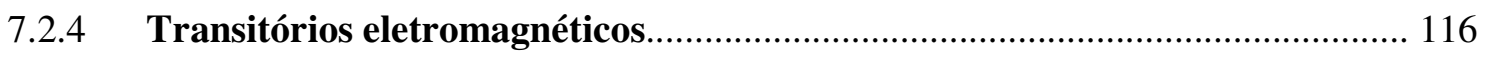

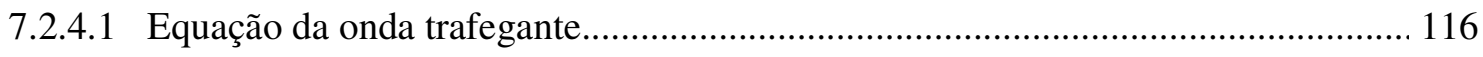

7.2.4.2 Comentários sobre a equação de onda tridimensional........................................ 119

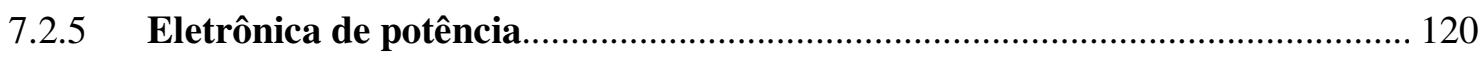

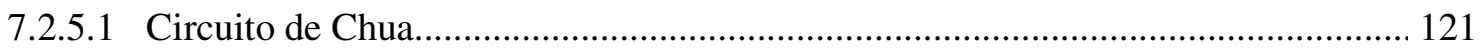

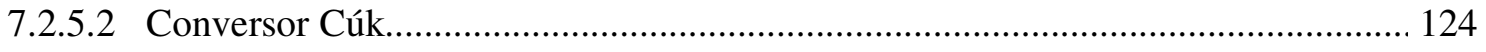

7.3 SOBRE O DETERMINISMO E AS IMPLICAÇÕES TEÓRICAS EM ENGENHARIA DE CONTROLE................................................................. 126

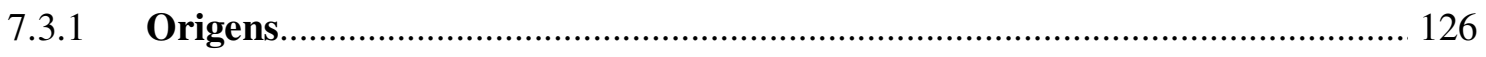

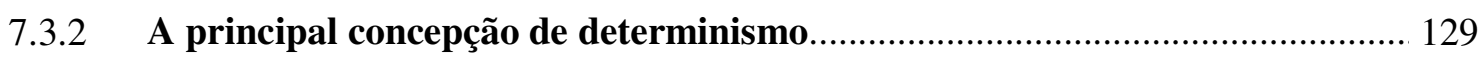

7.3.3 A influência do determinismo laplaciano...................................................... 130

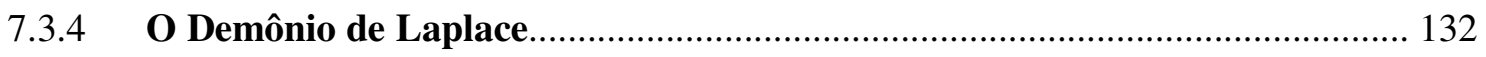

7.3.4.1 Características do "Demônio de Laplace"................................................................ 133

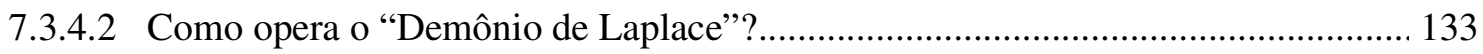

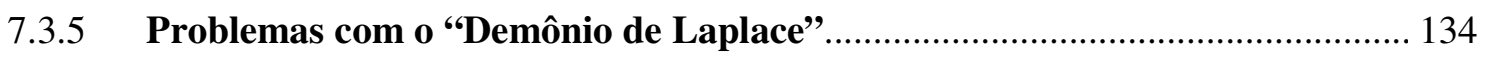

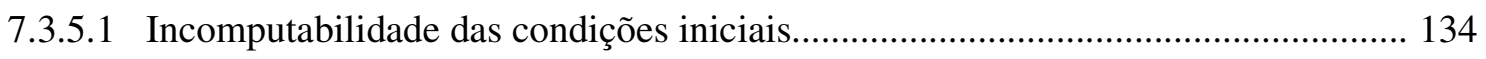

7.3.5.2 Incomputabilidade da solução das equações diferenciais..................................... 135

7.3.5.3 Incomputabilidade dos cálculos e de números em geral...................................... 136

7.3.6 O "Demônio de Laplace”’ e os sistemas caóticos............................................... 136

7.3.7 Considerações sobre causalidade e previsibilidade no contexto do "Demônio de Laplace"'................................................................................... 137

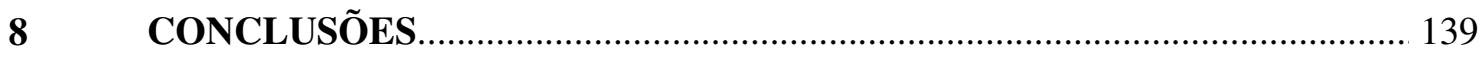

8.1 CONCLUSÕES SOBRE OS PROBLEMAS ABORDADOS DE SISTEMAS DE POTÊNCIA E ENGENHARIA DE CONTROLE..................................... 141

8.2 CRÍTICA AO CARTESIANISMO EM ENGENHARIA.................................... 143

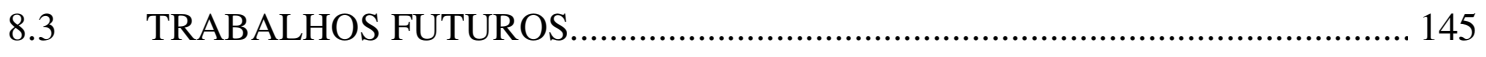

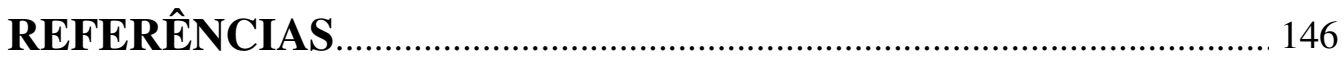

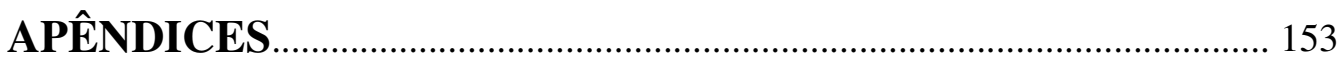

